\title{
Ensayo:
}

\section{Derechos colectivos de las comunidades sobre su propiedad intelectual: contradicciones entre propiedad industrial y conocimientos tradicionales}

\section{Resumen}

El presente ensayo tiene como objetivo el análisis de las contradicciones que se generan al interior del sistema de protección de la propiedad intelectual cuando este regula aspectos sobre conocimientos tradicionales y propiedad industrial. Se expresa el carácter comunitario de los conocimientos tradicionales en su estrecha relación con las comunidades y grupos sociales que los portan, lo que los hace de difícil encuadre en el sistema de propiedad industrial. Se refieren las características y esencia individual del sistema de propiedad industrial, que lo hacen incompleto a este tipo de saberes, lo que contribuye a la explotación y apropiación indebida por terceras personas ajenas a estas comunidades. Finalmente, se evidencia la importancia del reconocimiento de derechos colectivos a las comunidades sobre su propiedad intelectual en materia de conocimientos tradicionales.

\section{Palabras clave: conocimientos tradicionales, propiedad industrial, derechos colectivos.}

\begin{abstract}
The objective of this essay is to analyze the contradictions that arise within the intellectual property protection system when it refers to traditional knowledge and industrial property. The community cha-
\end{abstract}


racter of traditional knowledge is expressed in its close relationship with the communities and social groups that carry it, which makes it difficult to fit into the industrial property system. It refers to the characteristics and individual essence of the industrial property system, which make it incomplete for this type of knowledge and which contributes to the exploitation and misappropriation by third parties outside these communities. Finally, the importance of the recognition of collective rights to communities and social groups on their intellectual property in terms of traditional knowledge is evident.

Keywords: traditional knowledge, industrial property, collective rights. 


\section{Introducción}

Las normas jurídicas de propiedad industrial han sido empleadas por diversos grupos sociales tradicionales para proteger sus conocimientos y creaciones intelectuales en las actividades comerciales, tal es el caso de signos distintivos como las marcas, logos, emblemas, y denominaciones de origen. En muchos otros casos, la legislación de propiedad industrial ha sido utilizada en sentido contrario a los intereses de las comunidades para obtener titularidad de derechos sobre conocimientos tradicionales bajo sus principios clásicos occidentales, y de esta forma, despojar y robar conocimientos tradicionales a las comunidades cuando se ha pretendido emplear las normas de patentes de invención, para patentar "invenciones nuevas" sobre la base de un conocimiento tradicional colectivo, otorgándole la titularidad a un individuo ajeno a la comunidad. Hoy en día, se discute en numerosos espacios la conveniencia o no de la protección de los derechos de propiedad intelectual a los conocimientos tradicionales. Desde el 2000, existe un Comité Intergubernamental en el seno de la Organización Mundial de la Propiedad Intelectual (OMPI) que discute este asunto. Los debates se centran en el uso de la propiedad industrial, los derechos de autor y conexos, o el reconocimiento de derechos colectivos a las comunidades y grupos sociales tradicionales que han sido depositarios de estos conocimientos. En los últimos años, esta tendencia se ha ido abriendo paso en la comunidad científica a partir del carácter comunitario y colectivo que tienen estos saberes; sin embargo, aún hay legislaciones que no incorporan este tema a los nuevos temas de protección intelectual y otras, como la ecuatoriana, que lo acoge en un merecido y adecuado reconocimiento jurídico.

\subsection{Conocimientos tradicionales}

Es importante partir de definiciones reconocidas de conocimientos tradicionales y propiedad industrial para entender su simbiosis y contradicciones, en ese sentido:

Jhon Mugabe, citado por Yeney Acea en su artículo denominado "Bienes intelectuales de naturaleza sui generis: los conocimientos tradicionales del sector agrícola. Protección internacional", indica que:

Los conocimientos tradicionales corresponden al conjunto de todos los conocimientos y prácticas, explícitos o implícitos, que se utilizan en la gestión de las facetas socioeconómicas y ecológicas de la vida. Estos conocimientos se basan en las experiencias pasadas y en la observación, y suelen ser propiedad colectiva de una sociedad (Acea, 2015, pág. 58).

Por su parte, el Grupo Indígena Regional sobre Biodiversidad de la Comunidad Andina (CAN) citado por Rodrigo De la Cruz en su artículo denominado "Conocimientos Tradicionales, Biodiversidad y Derechos de la Propiedad Intelectual - Patentes", explica que:

“(...) Son todas aquellas sabidurías ancestrales y conocimientos colectivos e integrales que poseen los pueblos indígenas, afroamericanos y comunidades locales, fundados en la praxis milenaria y su proceso de interacción hombre-naturaleza, y transmitidas de generación en generación, habitualmente, de manera oral” (De la Cruz, 2010, pág. 77). 
De las definiciones expuestas, es necesario tomar en cuenta cuatro aspectos de suma importancia: 1) conocimiento y práctica a partir de la interacción hombre-naturaleza, que sirve para la 2) permanencia y desarrollo de la comunidad; 3) conocimientos y prácticas transmitidas de generación en generación por 4) vía oral.

En consecuencia, estos conocimientos y prácticas del hombre en interacción con la naturaleza están atravesados por una cosmovisión de complementariedad, de interconexión mutua, más no de explotación sobre ellos. Con respecto a la permanencia y desarrollo de la comunidad, es necesario tener en cuenta la totalidad social, en tanto que abarca a todos los miembros, sin exclusión, y es en tal sentido en el que los conocimientos y prácticas no son privatizados, pues caso contrario no tendrían sentido y perecería la comunidad como tal.

Para su permanencia y desarrollo, es necesario que los conocimientos y prácticas perduren en el tiempo, y es por tal motivo que se transmiten de generación en generación. Entre sus comunidades portadoras hay una concepción otrora del tiempo, en que se piensa en generaciones futuras. Y, por último, la transmisión por vía oral ha sido la práctica general, en tanto que el aprendizaje resulta desde los primeros años de vida de las personas, y por tal motivo, es el eje principal, un aprendizaje intergeneracional de abuelos, padres, hijos y nietos.

De esta forma se construyen los conocimientos y prácticas en las comunidades indígenas, siendo el ranti ranti (principio de reciprocidad) uno de los principios fundamentales en el quehacer indígena y, de una manera amena, se refuerza con mayor firmeza, el tejido social en la comunidad.

\subsection{Propiedad industrial}

Luego de ver qué se entiende por conocimientos tradicionales, pasemos a analizar la propiedad intelectual, y en particular, la propiedad industrial. Este término trata fundamentalmente de las normas jurídicas para la protección de las invenciones, las marcas de fábrica o de comercio, los diseños industriales, los modelos de utilidad, los lemas comerciales y la represión de la competencia desleal. Asimismo, comprende el derecho de autor que tiene como objeto las obras literarias, científicas y artísticas, y también otorga protección a los artistas, intérpretes y ejecutantes, a los productores de fonogramas y a los organismos de radiodifusión. Constituyen un sistema de derechos monopólicos reconocidos por el Estado, a los creadores, en principio, sobre sus creaciones durante un tiempo limitado, o a terceros, que pueden pasar a ser titulares de derechos patrimoniales (Lipszyc, 1998).

Pero, ¿cuál es el alcance de la propiedad industrial frente a los conocimientos tradicionales? En este caso, se vincula la protección de las legislaciones de propiedad industrial a lo que anteriormente definíamos como conocimientos tradicionales y que hoy ocupa la agenda de las preocupaciones de la OMPI por ser este otro de los temas asumidos por su Comité Intergubernamental sobre la Propiedad Intelectual, Recursos Genéticos, Conocimientos Tradicionales y Folklore, que está en estudio y para el cual ya existe un "Proyecto de objetivos políticos y principios fundamentales" (Organización Mundial de la Propiedad Intelectual, 2004, pág. 1), de protección de los conocimientos tradicionales. 
Con independencia de que los conocimientos tradicionales de los grupos y comunidades indígenas y tradicionales sean objeto de análisis en este momento por la OMPI, buscando una adecuada protección que salve las diferencias entre el sistema clásico occidental de propiedad industrial y las necesidades de las comunidades para detener la explotación indebida de los mismos a través de un sistema sui generis, lo cierto es que la legislación de propiedad industrial ha sido utilizada para proteger estos conocimientos, como es el caso de las normas de diseños industriales, marcas e indicaciones geográficas. En otros casos se han empleado conocimientos tradicionales como el lenguaje para distinguir productos y servicios de los grupos sociales, que tengan o no relación con sus culturas.

A decir de Espín Alba, las normas de propiedad industrial, citado por Pérez Peña, se utilizan para:

(...) proteger ante las formas falsas o que induzcan a error respecto a la autenticidad o el origen. En estos casos se citan la legislación de competencia desleal y la legislación relativa a marcas y otros signos distintivos. Los pueblos indígenas y tradicionales han registrado marcas de certificación para proteger la autenticidad y la calidad de sus artes y oficios lo que, a su vez, les aporta a sus producciones, un valor añadido (Pérez O. , 2012, pág. 106).

Ejemplos importantes en el contexto nacional, lo constituyen la marca Vispu (Vístete Puruwá), línea dedicada a la elaboración de atuendos o vestimentas con simbologías que identifican a la indumentaria del pueblo Puruwá y la marca Mushuc Runa, dedicada a la intermediación financiera. Estas dos marcas son representantes de las culturas indígenas de las provincias de Chimborazo y Tungurahua, respectivamente.

A estas comunidades les preocupa el hecho de que determinadas empresas comerciales no autorizadas tomen sus vocablos, nombres, diseños, símbolos y los utilicen y registren como marcas. Es frecuente que estos grupos sociales traten de prevenir usos de sus expresiones y obras que atenten contra afirmaciones falsas y engañosas de autenticidad y origen, o de omisiones en la declaración de origen, y contra el registro de signos y símbolos tradicionales como marcas comerciales, lo que ha sido calificado como protección defensiva por Wenland B. (Wendland, 2006).

La legislación de la propiedad industrial también ha sido empleada en múltiples ocasiones en sentido contrario o negativo a los intereses de las comunidades pues la modalidad de las invenciones o el derecho exclusivo de patente de invención (Zerda \& Forero), se ha utilizado para obtener derechos exclusivos sobre conocimientos tradicionales de comunidades por parte de entidades ajenas a estos grupos, obviando o burlando requisitos indispensables para su protección como la novedad o la actividad inventiva, al obtener el registro en territorios distintos del que tienen su sede estos conocimientos e incluso, donde se habla otro idioma, lo que es utilizado a su favor por los usurpadores de forma tal que impiden que las comunidades portadoras tengan una participación adecuada en los beneficios derivados de su utilización.

Somos del criterio de no conceder protección a los conocimientos tradicionales por la vía de las patentes de invención porque incluso, aun cuando se demuestre que no cumplan con los requisitos de patentabilidad, los procedimientos de oposición con posterioridad a la concesión de una patente 
pueden ser insuficientes, complicados y onerosos, especialmente para los países en desarrollo y, por ende, no resultar económicamente viables.

Se han producido muchas discusiones acerca de las contradicciones entre los conocimientos tradicionales y el derecho de propiedad industrial. Son innumerables los casos descritos de explotación de estos en los que se ha empleado la propiedad industrial en su versión más occidental, dirigida a la protección de resultados creativos individuales bajo modalidades como las patentes de invención y de diseños industriales que obvian que estas creaciones poseen un carácter colectivo en su esencia, pues están sujetas a fuertes relaciones colectivas donde, en muchos casos, resulta imposible identificar un creador individual, o porque se ha perdido en el tiempo, o porque el resultado no es de una sola persona, sino de miembros de toda la comunidad. Esto sin mencionar que gran parte de los conocimientos no constituyen creaciones susceptibles de protección por este sistema, como es el caso de los rituales, los juegos tradicionales u otras prácticas tradicionales.

Gran parte de la doctrina sobre los temas de propiedad intelectual (Haight Farley, 1997 y Acea Valdés, 2016) sostiene que los principios de las comunidades indígenas y tradicionales chocan con los principios del tradicional concepto de propiedad intelectual, en particular la propiedad industrial. Es este un terreno teórico en el que se aprecian las batallas ideológicas entre las posturas occidentales y las de las culturas indígenas a partir de su propia cosmovisión. Requisitos de protección que exigen las leyes de propiedad industrial para las patentes de invención como la "novedad" y la "aplicación industrial", resultan inconcebibles dentro de la cosmogonía indígena respecto a conocimientos tradicionales, donde estos conocimientos han sido desarrollados a partir de la satisfacción de necesidades propias de estos grupos, y no para su aplicación industrial a partir de si existe alguna creación similar con anterioridad, para rebasarla con motivo de obtener un derecho monopólico sobre ella, como es el caso de la protección de patente de invención. Es innegable el interés de las industrias occidentales sobre estos conocimientos como fuente de ingresos mercantiles, múltiples casos han sido documentados en el contexto latinoamericano, como el caso de la ayahuasca y la sangre de drago en Ecuador (León Calle, 2017).

El esquema de pensamiento occidental ha cosificado algo intangible: los conocimientos tradicionales de los pueblos indígenas originarios; y lo ha convertido en una nueva fuente de acumulación de capital, que implica, entre otras cosas, el desabastecimiento de los pueblos indígenas originarios en cuanto a los recursos naturales que ahí reposan y la pérdida de los conocimientos tradicionales. En ese sentido, se ha señalado que más del $90 \%$ de la diversidad biológica del mundo persiste en los bosques tropicales y subtropicales de América del Sur, Asia y África y que, más de 7000 compuestos medicinales de la medicina occidental han sido extraídos de las plantas, teniendo en cuenta los conocimientos tradicionales de sus comunidades sobre ellas, y siendo apropiados al procesarlos en productos, procesos o procedimientos mediante patentes por las multinacionales farmacéuticas o de alimentos (De Sousa Santos, 2003).

Cabe mencionar que las empresas trasnacionales "venden" una imagen de una vida armónica en las sociedades a partir del uso de los conocimientos tradicionales de los pueblos indígenas. Esta, no es más que una tergiversación del pensamiento y de vida de los pueblos indígenas, en tanto 
que estas ideas del hombre y de la naturaleza no tienen cabida en la cosmovisión de los pueblos indígenas; es decir, no hay división entre sujeto cognoscente y objeto cognoscible.

El patentamiento hace posible que la compañía monopolice el mercado de las nuevas variedades de plantas que se derivan de una planta original durante el término de la patente. El conocimiento tradicional, en ese caso, albergado dentro de las comunidades por años, reside en el conocimiento que tienen estas acerca de cuáles son las diferentes variedades de plantas y sus usos, así como qué técnicas se emplean mediante la combinación de ellas para sanar enfermedades o ser empleadas para obtener determinados resultados útiles al ser humano.

Para las compañías farmacéuticas, alimentarias, bancos de germoplasmas y más firmas biotecnológicas que están detrás de ellas, la potestad que tienen de patentar la diversidad biológica del mundo significa la promesa de nuevas e ingentes fuentes de ingresos económicos, o a los que hoy también se les conoce como la era del oro verde. Al respecto, la revista Information About Intellectual Property, No. 6, citada por Carlos Pérez Guartambel, en su libro titulado Justicia Indígena, informa:

(...) que la compañía Monsanto, por ejemplo, espera ganar 150 millones de dólares adicionales al año si logra patentar y llevar al mercado a uno de sus nuevos productos. Menciona que, una variedad de soya está diseñada para soportar las aplicaciones del herbicida Roundup, que el mismo Monsanto expende al mundo entero (Pérez C. , 2015, pág. 377).

El mismo autor, citando al profesor chileno Francisco Rothhamner, señala que "la ingeniería genética podría definirse como la manipulación directa del material hereditario, destinada a introducir determinantes nuevos deseables y suprimir a los indeseables" (Pérez C. , 2015, pág. 381). En este sentido, la idea de usufructo personal de estos conocimientos y creaciones es extraña a la visión de los pueblos indígenas. Pues toda creación que se produce dentro de un territorio indígena es un servicio y un aporte a la comunidad; $y$, consecuentemente, un aporte para la humanidad, esto en parte ha sido porque no han tenido circulante monetario en su medio y han tenido que ir buscando métodos para la solución de alguna enfermedad a través del uso de la flora y fauna que se encuentra en su hábitat.

He aquí donde surge la necesidad de proteger los conocimientos tradicionales, en tanto que se han tergiversado, dañando la vida de los pueblos indígenas y otros pueblos tradicionales, y no generando retribución justa y equitativa.

Los conocimientos tradicionales han sido utilizados por personas ajenas a la comunidad. Sus conocimientos han sido sustraídos de manera indebida por terceras personas que poco o nada han apreciado la forma de vida, como tampoco su hábitat, y que únicamente han sobrepuesto el ánimo de lucro por sobre el bienestar humano, esta vulneración de derechos ha sido ocasionada sobre todo por empresas trasnacionales que controlan el campo de la línea alimenticia, agrícola y farmacéutica, con la finalidad de generar mayores beneficios económicos en el mercado mundial, a pesar de la escasez de recursos naturales producto de la irresponsabilidad de las mismas empresas trasnacionales. Famosas han sido las denuncias ante casos de apropiación indebida de 
conocimientos tradicionales por trasnacionales que han obtenido patentes de invención basadas en estos, como el caso de la margosa y la cúrcuma en Brasil (Documento OMPI IP/C/W/370/Rev.1, 9/ 3 de 2006), las patentes en Estados Unidos y Europa de una proteína aislada de la brazeína, encontrada en una baya de África occidental utilizada desde tiempos ancestrales por los nativos (Grain, 2000) u otros como el caso del cactus de hoodia en Sudáfrica (IP/C/W/368/Rev.1, 8/2 2006).

El problema principal radica en que dichas empresas hacen uso de estos conocimientos tradicionales, lo protegen jurídicamente como si fuesen propios, pero sin generar regalías a la comunidad de origen, tanto por el uso de dichos conocimientos tradicionales, como por el uso de los recursos naturales que se encuentran en un territorio determinado.

Esta paradoja, con respecto a los recursos genéticos y conocimientos tradicionales, se debe según Donoso (2006), a una concepción errada sobre el carácter público de estos términos; es decir, que cualquier persona puede acceder a los mismos, sin autorización alguna. Este tema obedece a la consideración de que los conocimientos tradicionales están en el dominio público según el sistema clásico occidental de propiedad intelectual. Para el derecho, la institución jurídica de dominio público en la propiedad intelectual significa que los derechos sobre las creaciones expiraron luego de transcurrido el plazo fijado por la ley, lo que según las leyes y la doctrina clásica de propiedad intelectual transcurre a partir de diversos momentos, en los que se toma como punto de partida el momento de la creación, la fecha de la muerte del autor o la fecha de la solicitud de registro, con plazos que oscilan en derechos de autor la vida de este y 70 años post mortem autoris, 0 en la propiedad industrial, 2 años a partir de la solicitud de la patente. En conclusión, esta lógica aplicada a conocimientos tradicionales que se han pretendido proteger por un sistema clásico de propiedad intelectual resultan inaplicables, siendo entonces considerados en dominio público los mismos, y, por ende, susceptibles de uso libre porque se presume debido a su ancestralidad, que ya están en esa condición jurídica.

Donoso (2006) señala, además, que esta vulneración de los derechos de propiedad intelectual y acceso a los recursos genéticos proviene especialmente de grandes empresas trasnacionales, que en nombre de realizar un beneficio a la humanidad y fomentar el desarrollo económico de los países, lesionan dichos derechos. Es por este motivo que resulta necesario reemplazar la concepción pública de los conocimientos tradicionales con la concepción de dominio colectivo y comunitario, mediante el cual se reconocen derechos sobre los saberes y conocimientos tradicionales de propiedad intelectual bajo una nueva noción de derechos colectivos que desde el 2000 estudia la OMPI, y que reconoce en primer lugar la titularidad de derechos a las comunidades depositarias.

Respecto al uso de los medicamentos, "entre 1950 y 1980, el 25\% de las medicinas de receta vendidas en Estados Unidos se basaban en fármacos derivados de plantas. Mientras que, en la actualidad, el $80 \%$ de la población mundial utiliza estas medicinas" (Arias, 2005). La autora, Xiomara Arias, citando a The Heritage Foundation sobre el índice de Libertad Económica, demuestra que Ecuador se encuentra en los niveles más bajos de protección de los derechos de propiedad intelectual. A esto sustenta: 
"plantas patentadas en Estados Unidos, tales como la ayahuasca, la quinua, entre otras especies endémicas, que están generando regalías para laboratorios de origen extranjero, y por tal motivo, no genera ningún tipo de beneficio a las comunidades indígenas, no sólo con respecto al valor económico, sino también al valor cultural y tradicional (Arias, 2005, pág. 2).

Como vemos, uno de los objetivos de la protección de la propiedad intelectual, con respecto a los conocimientos tradicionales, es generar una retribución económica. En el mismo sentido, el Sistema Económico Latinoamericano y del Caribe (SELA) resume lo siguiente:

(...) los objetivos de la protección de los conocimientos tradicionales serían la exclusión de terceros al acceso y uso no autorizado, el control de su uso, la prevención del uso inadecuado e ilegal, el reconocimiento de los derechos colectivos, la distribución de beneficios y el mantenimiento y preservación de los conocimientos tradicionales (Sistema Económico Latinoamericano y del Caribe, 2009).

Esta protección es más consecuente con los intereses colectivos y el carácter comunitario de estos conocimientos, desarrollandose una reingeniería del sistema clásico de propiedad intelectual.

Por otra parte, Sara Charlotte Andreini (2013, pág. 14), en su tesis de pregrado denominado "La protección y control de los conocimientos tradicionales y la propuesta de un régimen sui generis. El Ecuador como caso de estudio", explica uno de los factores por el cual ha sido posible la apropiación indebida de los conocimientos tradicionales: "la noción que la biodiversidad y los recursos genéticos en particular, eran parte del patrimonio de la humanidad, es decir, pertenecían a todos y a nadie a la vez". Dicha noción era respaldada por los propios Estados, y es en tal medida, cómplice del robo de los conocimientos tradicionales en materia genética. Sin embargo, esta perdió validez continuamente a partir de la convención sobre Diversidad Biológica en 1992, en la cual se trata la importancia de la protección de los conocimientos tradicionales. Pero cabe recalcar que antes de dicha fecha, ya se realizaban apropiaciones indebidas de los conocimientos tradicionales y los recursos genéticos como se ha expresado en este trabajo, siendo denunciadas en foros internacionales.

\subsection{Derechos colectivos de propiedad intelectual sobre los conocimientos tradicionales}

Los conocimientos tradicionales definen a las sociedades indígenas y tradicionales. Son actividades, actuaciones, modos vivos, realizados o practicados por estas, y es por tal razón que forman parte de un patrimonio colectivo. Son el resultado de un proceso social y cultural, por ende, su titularidad es comunitaria, lo que implica que cualquier decisión que se vaya a tomar sobre los mismos, se debe realizar con la participación de toda la comunidad a través de sus instituciones de representación. En una posible exploración, desarrollo o utilización de conocimientos tradicionales por parte de un agente externo es importante el beneficio colectivo.

Es así que se debe entender que no son bienes de dominio público. Estos conocimientos le pertenecen a los miembros de las comunidades que los generaron, quienes deben autorizar el uso, acceso o aprovechamiento de tales recursos. Este último detalle es similar al sistema clásico de propiedad intelectual que reconoce derechos patrimoniales, lo que la condición de sus titulares 
es diferente, no es un individuo, sino un colectivo, que tiene derecho a participar de manera justa y equitativa de los beneficios obtenidos.

En el orden positivo, y en la búsqueda de un sistema de protección ideal para los conocimientos tradicionales, en el año 2000 se ha creado el Comité Intergubernamental de la OMPI sobre la Propiedad Intelectual y Recursos Genéticos, Conocimientos Tradicionales y Folclore (CIG), que todavía en el año 2020, no ha dicho la última palabra sobre la protección de los conocimientos tradicionales por la propiedad intelectual.

Este comité ha examinado las propuestas de diversos países y las experiencias nacionales en torno a esta protección. Diversas han sido las experiencias de protección que se han tratado de implementar, buscando una protección adecuada para los conocimientos tradicionales. Varios son los casos en que se ha buscado la posibilidad de reconocer derechos de propiedad intelectual de manera diferente al sistema tradicional de propiedad intelectual. Siguiendo este orden y mucho antes del Comité de la OMPI., "a partir de 1992, al celebrarse la Cumbre de la Tierra en Río de Janeiro, se pudieron reconocer dos tipos de posiciones relacionadas con los derechos de propiedad intelectual de los pueblos indígenas" (Álvarez, 2006, págs. 58-59):

- La que sostiene que se debe reconocer algunos aspectos del régimen de propiedad intelectual basados en la comercialización, pero dentro del marco de reconocimiento de propiedad colectiva de los conocimientos, la protección del medio ambiente, la promoción de regímenes cooperativos y el reconocimiento del beneficio para los depositarios indígenas.

- La segunda, que rechaza el régimen de propiedad intelectual occidental que consagra los derechos individuales, lo acusa de constituir una amenaza a los derechos colectivos de las poblaciones nativas y vincula la necesaria protección al patrimonio cultural y al territorio. Este punto de vista es apoyado por la Coordinadora de Organizaciones Indígenas de la Cuenca Amazónica (COICA).

Respecto a los aspectos de propiedad intelectual de las comunidades, en la actualidad se aplican varios sistemas sui generis, que han tomado como referente en un inicio la Ley sobre Derecho de Autor de 1976 y las disposiciones tipo OMPI/UNESCO de 1982, y que difieren de las tradicionales de propiedad intelectual. Resulta interesante el hecho de que en casi la totalidad de estos casos se establece como titulares de los derechos la comunidad o comunidades tradicionales que cultivan estas expresiones y conocimientos, así como el establecimiento de autoridades competentes que otorgan o no la autorización de uso, o el consentimiento fundamentado previo para ello. Para ello, la OMPI, a través del CIG, en su quinta sesión realizada en Ginebra de 7 al 15 de julio de 2003, señaló:

(...) el régimen especial de Propiedad Intelectual sobre los Derechos Colectivos de los Pueblos Indígenas, para la protección y Defensa de su Identidad Cultural y de sus Conocimientos Tradicionales de 2000, de Panamá y su correspondiente Decreto Ejecutivo de 2001; y el Marco Regional para el Pacífico Meridional relativo a la protección de los conocimientos tradicionales y las expresiones de la cultura de 2002 (OMPI, 2003). 
En el primer caso se establece un sistema de registro para los conocimientos tradicionales. Se ha creado un despacho especial dentro de la oficina de propiedad intelectual del país encargado de aprobar las solicitudes y mantener el registro. Las formalidades que hay que seguir ante la oficina de la propiedad intelectual no requieren los servicios de un abogado y no existen tasas que graven las solicitudes. Se reconocen derechos colectivos exclusivos frente a terceros para prevenir distorsión y alteración, derechos patrimoniales de reproducción y de explotación; en todos los casos, respecto al tiempo, se establece que es indefinido. A este sistema se le critica el hecho de que sólo cubre situaciones ante usos comerciales, por ejemplo "los derechos no son aplicables cuando los conocimientos tradicionales nacionales son utilizados por entidades públicas para usos no comerciales" y la protección de los conocimientos tradicionales que no tienen uso comercial es necesaria y significativa también para las comunidades. Además, el sistema de Panamá requiere el registro de los conocimientos y expresiones "lo que podría derrotar el propósito de la protección especialmente respecto a conocimientos y expresiones que son sagradas o secretas" (Raorane, 2006).

En el segundo caso, los "propietarios tradicionales" o la autoridad encargada de asuntos culturales tienen el derecho a autorizar o impedir, entre otras cosas, la adaptación, transformación o modificación de las expresiones culturales y conocimientos tradicionales protegidas. Los usuarios externos deben estar autorizados si quieren hacer una obra derivada. Cualquier derecho de propiedad intelectual sobre las obras derivadas recae sobre el autor de la obra, pero si la obra se utiliza con fines comerciales, el titular de los derechos debe repartir los beneficios con los propietarios tradicionales, reconocer la fuente de la expresión cultural o conocimiento tradicional y respetar los derechos morales sobre dichas expresiones.

Respecto al término de duración, se reconoce la perpetuidad de los mismos. En este caso, se observa además, cómo quedan regulados los derechos ante utilizaciones con fines comerciales sin velar con cuidado aquellos usos que se hagan sin fines comerciales y que quedan incluidos en la ley dentro de limitaciones, como los usos educativos o de ilustraciones que son preocupantes en los casos de conocimientos sagrados o secretos para las comunidades.

Apartándose de la línea comercial, algunos autores reconocen la fuerza del derecho consuetudinario para las comunidades como una alternativa válida para resolver los conflictos ante violaciones que se produzcan de sus conocimientos tradicionales. Para Raorane (2006), una aplicación eficaz de ley de costumbre indígena para la protección de conocimientos tradicionales puede lograrse con soluciones tales como educar a terceros sobre las leyes de costumbre indígenas.

Un mecanismo ilustrativo sería crear una presunción de que el uso de conocimientos tradicionales por terceros es desautorizado a menos que la autorización explícita se conceda por la comunidad que posee el conocimiento tradicional. Así, sólo se esperará que un usuario de un conocimiento tradicional investigue la ley particular para el uso deseado y se relevará el aprendizaje sobre todas las leyes de costumbre indígenas potencialmente aplicables. Por ejemplo, en Australia, las fiestas que desean usar el arte aborigen tendrían la carga de obtener la autorización explícita de la comunidad a que la obra de arte pertenece. Así, ellos tendrían que estar conforme a ambas leyes, la ley de costumbre indígena, así como la ley de propiedad intelectual australiana. Los 
Estados también pueden crear programas de educación para diseminar la información sobre los procesos apropiados para el uso de conocimientos tradicionales (Raorane, 2006). Al respecto, en la realidad social y jurídica australiana se vienen recogiendo estas enseñanzas desde hace años debido al choque del sistema clásico de propiedad intelectual y su inoperancia ante conocimientos tradicionales, lo que ha dirigido la atención hacia la pertinencia de una legislación sui generis para ese país.

Álvarez Navarrete, en su libro titulado: Derecho de ¿autor? El debate de hoy, señala que, si bien son valoradas tanto la posibilidad de reconocimiento de derechos colectivos o de derecho consuetudinario, todavía hay organizaciones internacionales y determinados países como la Organización Mundial del Comercio (1994) y Estados Unidos que enturbian estas posibilidades.

Al respecto, la OMPI argumenta que sui generis se emplea para describir un régimen concebido para proteger los derechos que no están contemplados en las doctrinas tradicionales de patentes, marcas, derecho de autor y secreto comercial (OMPI, 2019). En este sentido, y tomando en cuenta que se va a normar un derecho colectivo vinculado al principio de libre determinación o autodeterminación, se debe agrupar en el sistema de protección de los conocimientos tradicionales elementos que componen los derechos intelectuales.

Lo cierto es que, actualmente, en América Latina ya son varios los países que se acogen al sistema de protección sui generis de propiedad intelectual: Ecuador, Panamá, Bolivia y Venezuela, para proteger a los conocimientos tradicionales al reconocer derechos colectivos de propiedad intelectual a las comunidades. En ese orden, limitan el clásico sistema de propiedad intelectual (derechos de autor y conexos, y derechos de propiedad industrial) que favorece a los creadores individuales siempre que se pretenda establecer titularidad de derechos de propiedad intelectual de conocimientos tradicionales en detrimento de sus verdaderos portadores, en este caso, las comunidades indígenas, tradicionales o grupos sociales que han ido recreando tradicionalmente sus conocimientos.

\section{Para Pérez Peña:}

En la actualidad se ha desarrollado un sistema sui generis de protección con el que se identifican en mayor medida determinadas comunidades para la protección de sus derechos intelectuales. Este sistema difiere del de derecho de autor y lentamente ha ido estableciéndose tanto por la doctrina, como por la práctica cultural y la legislación nacional. Este sistema opera sobre la base del reconocimiento de derechos colectivos y del pluralismo jurídico latinoamericano, al ratificar la esencia comunitaria de estas obras y expresiones, y la titularidad de los derechos se sitúan en cabeza de las propias comunidades portadoras. Varias leyes nacionales de los países estudiados apuestan por este sistema, como es el caso de la venezolana, solo en el texto constitucional, la panameña y la ecuatoriana (Pérez Peña, 2018).

El Ecuador había reconocido desde 1998, en su Constitución, el carácter colectivo de estos derechos (artículo 83 apartado 9 ,Constitución Política de la República del Ecuador, 1998). En la Constitución de 2008, vigente en este momento, en su artículo 322 se declara que:

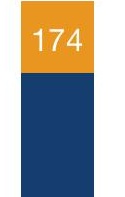


Se prohíbe toda forma de apropiación de conocimientos colectivos, en el ámbito de las ciencias, tecnologías y saberes ancestrales. Se prohíbe también la apropiación sobre los recursos genéticos que contienen la diversidad biológica y la agro-biodiversidad (Constitución de la República del Ecuador, 2008).

Con lo que queda legitimado constitucionalmente este sistema sui generis. Y luego en el artículo 402, establece que:

Se prohíbe el otorgamiento de derechos, incluidos los de propiedad intelectual, sobre productos derivados o sintetizados, obtenidos a partir del conocimiento colectivo asociado a la biodiversidad nacional (art.402, Constitución de Ecuador, 2008).

Con estos dos artículos se regula, en el ámbito constitucional nacional, el reconocimiento de la titularidad de los derechos colectivos de propiedad intelectual a favor de las comunas, comunidades, pueblos y nacionalidades indígenas, afroecuatorianos y montubios del Ecuador.

A partir del año 2016, con la promulgación del Código Orgánico de la Economía Social de los Conocimientos, la Creatividad y la Innovación, se establece el procedimiento administrativo para la protección de estos conocimientos por la vía de los derechos de propiedad intelectual, y el reconocimiento que poseen en la generación de conocimiento colectivo a partir de una distribución equitativa y democrática que permite su acceso.

Son regulados en este código diversos temas asociados a su protección y gestión como: formas de protección (art. 522), depósito voluntario por parte de las comunidades (art.523), custodia en el ámbito comunitario (art. 524), acceso, uso y aprovechamiento indebidos (art.525), solicitudes y concesión o negativa (arts. 529 y 531), contratos y registro (arts.532 y 533), solución de controversias (art. 534), sanciones (art.535) y gestión (art. 537).

En todos los casos, la normativa reconoce las facultades de los grupos comunitarios respecto a sus conocimientos tradicionales en materia de propiedad intelectual, y la imposibilidad de establecer un sistema de protección jurídica individual de propiedad industrial sobre dichos conocimientos sin el consentimiento de sus legítimos titulares, las comunidades, y sin una distribución equitativa de los dividendos derivados, en aquellos casos que estos autorizasen su explotación en modalidades de propiedad industrial.

\section{Conclusiones}

La propiedad industrial es una de las ramas de la propiedad intelectual, por tanto, bajo esta concepción los conocimientos tradicionales, es una subcategoría de creaciones asociadas a los derechos intelectuales.

Diversas son las contradicciones entre el derecho de propiedad industrial y los conocimientos tradicionales. Los principios de la propiedad industrial clásica se contraponen con la naturaleza de las creaciones producidas por las comunidades, pueblos y nacionalidades indígenas, por lo que el sistema legal, para proteger a los conocimientos tradicionales, debe ser una herramienta 
autónoma, independiente, sui generis, como ya muestran varios países a nivel internacional y en el ámbito regional latinoamericano.

El entendimiento de que los conocimientos tradicionales pertenecen a los grupos que durante siglos los han recreado en un proceso vivo y activo, a partir de su naturaleza colectiva, supone en el orden jurídico asumir que en materia de propiedad intelectual los derechos sobre estos son derechos colectivos que deben ser reconocidos a estos grupos sociales.

El Ecuador ha reconocido constitucionalmente y en legislación especial estos derechos en su esencia comunitaria, evidenciando la necesidad de contar con las comunidades y grupos sociales, antes de atribuir cualquier derecho individual sobre alguna modalidad de propiedad industrial relacionada a conocimientos tradicionales.

Este reconocimiento permite disminuir considerablemente las asimetrías o contradicciones entre el sistema de propiedad industrial y los conocimientos tradicionales en cuanto a su protección y gestión. En mi opinión, el legislador ha optado por establecer un sistema que está acorde con las necesidades de las comunidades indígenas y sus derechos, en torno a su conocimiento tradicional, adelantándose incluso a la esperada, hace 20 años, normativa internacional que, en el caso del derecho internacional público, establezca derechos y obligaciones para los estados parte que la suscriban. De igual forma, con ello se trata de proteger a estos grupos sociales ante las prácticas indebidas que se suscitan en el contexto nacional y que, en muchos casos, poseen causas diversas, derivadas en su complejidad de fenómenos como la globalización y donde el derecho y la vida de las comunidades respecto a su cosmogonía también resulta estar inmersa. 


\section{Referencias}

Acea, Y. (2015). Bienes intelectuales de naturaleza sui generis: los conocimientos tradicionales del sector agrícola. Protección internacional. 17(22), 53. https://revistas.ucc.edu.co/index.php/di/article/view/1242

Acea Santos, Y. (2016). Una mirada a la protección de los conocimientos tradicionales agrícolas desde el contexto latinoamericano. YACHANA, 5(1), 113-120. http://revistas.ulvr.edu.ec/index.php/yachana/article/ view/288

Acea Santos, Y. (2016). El sistema de propiedad intelectual y la protección de los conocimientos tradicionales. Apuntes necesarios. Revista Alegatos., número 94, México septiembre-diciembre. http://revistastmp.azc. uam.mx/alegatos/index.php/ra/article/view/5

Álvarez, L. (2006). Derecho de ¿autor? El debate de hoy. La Habana, Cuba: Ciencias Sociales. http://up-rid2. up.ac.pa:8080/xmlui/handle/123456789/1867

Andreini, S. (2013). La protección y control de los conocimientos tradicionales y la propuesta de un Régimen Sui Generis. El Ecuador como caso de estudio (tesis de pregrado). Quito, Ecuador: Universidad San Francisco de Quito.

Arias, X. (2005). La Propiedad Intelectual: Enfoques para el Tratamiento Económico de los Conocimientos Tradicionales. Quito: Pontificia Universidad Católica del Ecuador.

Comité Intergubernamental OMPI, WIPO/GRTKF/IC/10/INF/5(a). (2006). Panel de la OMPI sobre las "comunidades locales e indígenas" preocupaciones y experiencias en la promoción, el matenimiento y la protección de sus conocimientos tradicionales, de sus expresiones culturales tradicionales y de sus recursos genéticos. OMPI, Comité Intergubernamental sobre Propiedad Intelectual y Recursos Genéticos, Conocimientos Tradicionales y Folklore, Ginebra.

Comité Intergubernamental sobre Propiedad Intelectual y Recursos Genéticos, Conocimientos Tradicionales y Folclore. (2000). Asuntos relacionados con la propiedad intelectual, los recursos genéticos, los conocimientos tradicionales y el folklore. Ginebra: Organización Mundial de la Propiedad Intelectual.

De la Cruz, R. (2010). Conocimientos Tradicionales, Biodiversidad del Ecuador y Derechos de la Propiedad Intelectual-Patentes. AFESE 54, 77-96. https://afese.com/img/revistas/revista54/ddpropiedad.pdf

De Sousa Santos, Boaventura. (2003). La Caída del Angelus Novus: Ensayos para una nueva teoría social y una nueva práctica política. Bogotá: ILSA, 146

Documento OMPI IP/C/W/370/Rev.1, 9/3 de 2006.

Documento OMPI IP/C/W/368/Rev.1, 8/2 2006. La relación entre el Acuerdo sobre los ADPIC y el Convenio so- 
bre la Diversidad Biológica resumen de las cuestiones planteadas y las observaciones formuladas

Donoso, S. (2006). Propiedad Intelectual: recursos genéticos, conocimientos tradicionales y folklor. Hacia la creación de un sistema sui géneris de propiedad intelectual. Quito: Impresoresmyl.

GRAIN. (2000). De patentes y piratas: EI ABC del patentamiento de la vida. Montevideo

Ecuador, Asamblea Constituyente. (2008). Constitución de la República del Ecuador. Quito: Registro Oficial N 449 de 20 de octubre de 2008.

Ecuador, Asamblea Nacional. (2016). Código Orgánico de la Economía Social de los Conocimientos, Creatividad e Innovación. Quito: Registro Oficial Nro. 899 de 09 de diciembre de 2016.

Ecuador, Congreso Nacional. (1998). Artículo 83 apartado 9, Constitución Política de la República del Ecuador. Quito: Registro Oficial Nro. 1 de 11 de agosto de 1998.

Ecuador, Congreso Nacional. (1998). Constitución Política de la República del Ecuador. Quito: Registro Oficial Nro. 1 de 11 de agosto de 1998.

Ecuador, Congreso Nacional. (1998). Ley de Propiedad Intelectual. Quito: Registro Oficial Nro. 320 de 19 de mayo de 1998.

Ecuador, Congreso Nacional. (2006). Ley de los derechos colectivos de los pueblos negros o afroecuatorianos. Quito: Registro Oficial Nro. 275 de 22 de mayo de 2006.

Haight Farley, C. (1997). Protecting folklore of indigenous peoples is intellectual property the answer. Connecticut Law Review. Consultado el 15 de marzo de 2020: https://heinonline.org/HOL/LandingPage?hand-

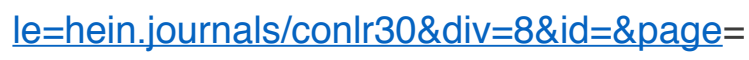

León Calle, S. (2017). La protección de los conocimientos tradicionales en el ordenamiento jurídico de propiedad intelectual internacional y ecuatoriano. ¿Empoderamiento o regularización? Deusto Journal of Human Rights, España. https://doi.org/10.18543/djhr-2-2017pp49-70

Lipszyc, D. (1998). Derecho de Autor y derechos conexos. Zavalia: UNESCO.

Organización Mundial del Comercio. (1994). Acuerdo sobre los Aspectos de los Derechos de Propiedad Intelectual relacionados con el Comercio.

Organización Mundial de la Propiedad Intelectual. (2003). Resumen comparativo de la legislación sui generis para la protección de las expresiones culturales tradicionales. OMPI, Comité intergubernamental sobre propiedad intelectual y recursos genéticos, conocimientos tradicionales y folclore, Ginebra.

Organización Mundial de la Propiedad Intelectual. (2004). Proyecto de objetivos políticos y principios funda- 
mentales: informaciones generales y deliberaciones previas. Ginebra, Suiza. Recuperado el 25 de julio de 2019.

Organización Mundial de la Propiedad Intelectual. (2019). Propiedad intelectual y conocimientos tradicionales. Ginebra, Suiza.

Pérez, C. (2015). Justicia Indígena. (Tercera edición ed.). Quito.

Pérez Peña, O. (2012). Protección de las obras de la cultura popular tradicional por el derecho de autor: especial referencia a Cuba (tesis doctoral). La Habana.

Pérez Peña, O. (2018). Derecho de Autor y cultura popular tradicional en América Latina y el Caribe. La propiedad inmaterial, 27-48. https://revistas.uexternado.edu.co/index.php/propin/article/view/5516

RaoRane, M. (2006). Aiming Straight: The Use of Indigenous Customary Law to Protect Traditional Cultural Expressions en Pacific Rim Law and Policy Journal, September. 15Pac.RimL. \& Pol'y J.827. Disponible en: https://digitalcommons.law.uw.edu/wilj/vol15/iss3/8 consultado 27 de marzo de 2020

Sistema Económico Latinoamericano y del Caribe. (2009). La protección de los conocimientos tradicionales, las expresiones culturales tradicionales (folklor) y los recursos genéticos conexos: Enfoque desde el SELA. WIPO, Caracas.

Wendland, W. B. (2006). Intellectual property and the protection of traditional knowledge and Cultural expressions. In "Art and Cultural Heritage: law, policy, and practice" (pp. p-327).

Zerda, Á., \& Forero, C. (s.f.). Los derechos de propiedad intelectual sobre los conocimientos de las comunidades étnicas. Recuperado el 25 de julio de 2019 de http://www.cerlalc.org 
Copyright (c) 2021 Oscar Alberto Pérez Peña

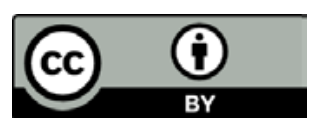

Este texto está protegido bajo una licencia internacional Creative Commons 4.0.

Usted es libre para Compartir-copiar y redistribuir el material en cualquier medio o formato - y Adaptar el documento - remezclar, transformar y crear a partir del material-para cualquier propósito, incluso para fines comerciales, siempre que cumpla las condiciones de Atribución. Usted debe dar crédito a la obra original de manera adecuada, proporcionar un enlace a la licencia, e indicar si se han realizado cambios. Puede hacerlo en cualquier forma razonable, pero no de forma tal que sugiera que tiene el apoyo del licenciante o lo recibe por el uso que hace de la obra.

\section{$\underline{\text { Resumen de licencia - Texto completo de la licencia }}$}

